\title{
Offloading Intervention in Healing of Diabetic Foot Ulcers: A Narrative Review
}

\author{
Shraddha Modi ${ }^{1}$, Tirou Aroul Tirougnanassambandamourty ${ }^{2}$
}

\begin{abstract}
Today, diabetic foot ulcers (DFUs) are responsible for most hospitalizations compared to any other complication of diabetes. Foot ulceration is common and affects up to $25 \%$ of the diabetic population during their lifetime, resulting in the most common cause of hospitalizations ( 30\%). Plantar ulcers are the commonest neuropathic lesions in diabetes patients due to unrecognized trauma and chronic hyperglycemia are some of the known risk factors. In this review article, we assess the healing ability of DFUs if an offloading intervention was used.

Keywords: Diabetic foot ulcers, Diabetic peripheral neuropathy, Peripheral vascular disease.

Annals of SBV (2021): 10.5005/jp-journals-10085-8133
\end{abstract}

\section{INTRODUCTION}

The foot being a complex structure is the foundation for the whole body. ${ }^{1}$ Diabetic foot as defined by the World Health Organization is, "The foot of a diabetic patient that has the potential risk of pathologic consequences, including infection, ulceration, and/or destruction of deep tissues associated with neurologic abnormalities, various degrees of peripheral vascular disease, and/or metabolic complications of diabetes in the lower limb". Foot ulceration is common and affects up to $25 \%$ of the diabetic population during their lifetime. The offloading intervention of foot ulcers with pad/cast surrounds the ulcer and provides it a cushion-like effect and offloads it from the body weight. Therefore, it becomes essential to use a cost-saving technique that can reduce the diabetic foot ulcer (DFU) burden on diabetes patients for a developing country like ours which contributes to the major global burden of DFUs.

\section{Materials and Methods}

An electronic search strategy was used to select the studies from different databases like PubMed, PubMed Central, Google Scholar, and Research Gate. A combination of the keywords epidemiology of DFUs, etiopathogenesis of DFUs, diabetic peripheral neuropathy, macro-angiopathy, and offloading interventions was used to select the articles. A detailed review was done among the results from the extensive search and from a few of the recent articles which were more precise to our topic of interest.

\section{Etiopathogenesis of Diabetic Foot Ulcer}

Involuntary mechanical changes in the conformation of the bony architecture of the foot, peripheral neuropathy, and atherosclerotic peripheral arterial disease, occur in higher frequency and intensity in the diabetic population. ${ }^{2}$ The risk factors for DFU are as follows: gender (male), duration of diabetes longer than 10 years, and other comorbidities. ${ }^{3}$ Uncontrolled diabetes mellitus (DM) exaggerates the development of neuropathy and peripheral arterial disease, loss of sensation of the feet, and ischemia, a combination of which leads to foot ulcers. ${ }^{4}$ Diabetes is associated with Charcot's arthropathy, which results in progressive destruction of the bones, joints, and

\footnotetext{
${ }^{1}$ Department of General Surgery, Mahatma Gandhi Medical College and Research Institute, Gulbarga, Karnataka, India

${ }^{2}$ Department of General Surgery, Mahatma Gandhi Medical College and Research Institute, Puducherry, India

Corresponding Author: Shraddha Modi, Department of General Surgery, Mahatma Gandhi Medical College and Research Institute, Gulbarga, Karnataka, India, Phone: +91 9008445394, e-mail: shraddha. modi1310@gmail.com

How to cite this article: Modi S, Tirougnanassambandamourty TA. Offloading Intervention in Healing of Diabetic Foot Ulcers: A Narrative Review. Ann SBV 2021;10(1):12-14.

Source of support: Nil

Conflict of interest: None
}

soft tissues and is commonly seen in the ankle and foot. In countries with lower socioeconomic status barefoot walking and lack of foot care awareness are a few of the factors that increase the burden of foot disease. ${ }^{4}$

\section{Diabetic Peripheral Neuropathy}

Diabetic peripheral neuropathy (DPN) is a complication seen in patients with DM, also affecting $50 \%$ of patients with type 1 diabetes. Waaijman et al. in a prospective study of risk factors for recurrence of the plantar diabetic ulcer in DPN patients concluded that the presence of a minor lesion was fairly the strongest predictor and use of adequate offloaded footwear was the strong protector against ulcer recurrence from unrecognized repetitive trauma. ${ }^{5}$

\section{Diabetes-related Atherosclerosis}

Diabetes-related atherosclerosis is present in the proximal arteries of the limb and manifests as iliac, femoral, and popliteal disease. Other risk factors are hypercholesterolemia and smoking. ${ }^{6}$ Mozes et al. analyzed amputated legs of diabetic patients and concluded that diabetic patients are more likely to develop calcification in tunica media causing more severe occlusive disease. ${ }^{7}$ Clinically, they present with intermittent claudication of the buttocks or the calf region. ${ }^{8}$ 


\section{Diabetic Foot Infection (DFI) and Osteomyelitis}

Sixty percent of DFUs are secondarily complicated by infection and in two-third of the cases is responsible for major lower limb amputations. ${ }^{9}$ Gram-positive Staphylococcus aureus is most often involved in DFI complicating both neuropathic and ischemic ulcers. Diabetic foot osteomyelitis (DFO) is the consequence of a soft tissue infection that spread into the bone, involving the cortex first and then the marrow..$^{10}$ Osteomyelitis is a common DFI seen in $10-15 \%$ of moderate and $50 \%$ of severe infections. Bone involvement is suspected in all patients with DFU, patients with secondary infection, chronic wounds, and recurrent ulcers.

\section{Prevention Strategies}

Prevention is the first step toward the diabetic foot. Foot ulceration is common and affects up to $25 \%$ of the diabetic population during their lifetime. Strategies aimed at preventing foot ulcers by surveillance on the patients with recognized risk factors are cost-effective and are more beneficial when patient education is prioritized and lifestyle modification such as cessation of smoking. A meta-analysis conducted by Ragnarson et al. showed that smoking harms the wound healing of diabetic foot individuals. ${ }^{11}$ Timely and effective management of DFU can reduce preventable amputations and its associated mortality and quality of life. The management of DFU with a multidisciplinary team consists of a general practitioner, a nurse, an educator/counselor, an orthotic specialist, a podiatrist, endocrinologists, dieticians, and orthopedic specialists. A strict glycemic control wound debridement, offloading modalities, and advanced dressings are a part of DFU management. ${ }^{12}$ Advanced therapies like hyperbaric oxygen therapy, electrical stimulation, and negative pressure wound therapy, bio-engineered skin, and growth factors are used as adjunctive for rapid healing of DFU. ${ }^{13}$ The forefoot is more prone to develop infections due to diabetic neuropathy which alters the structure of the foot leading to the formation of new pressure points on the toes and metatarsal heads which are more susceptible to mechanical trauma. Sutkowska et al. in their study interpreted that the forefoot is the most frequent region of the foot which bears the highest pressure on the sole especially in patients with diabetes it is the central part of the forefoot the 2nd and 3rd metatarsal heads. ${ }^{14}$ These ulcers are sometimes known as hard-to-heal ulcers which usually respond to advanced treatment and offloading interventions.

\section{Offloading Interventions}

Uncomplicated plantar ulcers usually heal within a duration of 6-8 weeks with adequate offloading are considered chronic if no clinical improvement occurs during 3 months of various offloading treatments are present for hard to heal plantar DFUs such as total contact cast (TCC), removable contact cast (RCC), customized therapeutic footwear, shoe model cast (SMC), etc. In several studies which were analyzed TCC was found to be the most effective form to off-load an ulcer. However, complications such as infections, maceration were not significant, patient compliance, and cost analysis could be a major drawback. As patients with TCC experience difficulty in adherence to it. In this period of challenging economic background, the need for more cost-effective offloading devices which can be used by all the classes of patients irrespective of their economic background for the healing of DFU should be made available. Faglia et al. and Piagessi et al. in their studies had a similar conclusion and stated that TCCs are the most expensive offloading devices as the materials used in them cannot be reused and, it requires medical supervision and is applied by qualified professionals. ${ }^{15}$ Cavanagh and Bus in their study mentioned that casts are the best device to off-load a plantar ulcer and must be used as the first line of treatment. Other devices such as customized therapeutic footwear, accommodative dressings, or modified insoles are used to prevent ulcers in patients with diabetic neuropathy, and also as a second-line treatment in healed ulcers to prevent a recurrence. ${ }^{16}$

\section{Conclusion}

Summarizing the review, if keen surveillance was maintained on patients with recognized risk factors and comorbidities for the development of foot problems followed by a regular visit to the foot clinic once a trimester and adequate offloading the foot we can help reduce the disease burden. We would like to recommend the use of offloading dressing for up to 2 weeks or more depending on the size, depth, and extent of the ulcer followed by the use of conventional dressing with customized footwear/insoles and carries high value in preventing ulcer recurrence.

\section{References}

1. Kavitha KV. Choice of wound care in diabetic foot ulcer: a practical approach. World J Diabetes 2014;5(4):546. DOI: 10.4239/wjd.v5.i4.546.

2. Diabetic Ulcers: Practice Essentials, Pathophysiology, Etiology. 2020 Jan 22 [cited 2020 Feb 2]; Available from: https://emedicine. medscape.com/article/460282-overview\#a3.

3. Monteiro-Soares M, Boyko EJ, Ribeiro J, Ribeiro I, Dinis-Ribeiro M. Predictive factors for diabetic foot ulceration: a systematic review: Predictive factors for diabetic foot ulceration. Diabetes Metab Res Rev 2012;28(7):574-600. DOI: 10.1002/dmrr.2319.

4. Mishra SC, Chhatbar KC, Kashikar A, Mehndiratta A. Diabetic foot. BMJ 2017;359:j5064. DOI: 10.1136/bmj.j5064. Available from: https:// www.bmj.com/content/359/bmj.j5064.

5. Waaijman R, de Haart M, Arts MLJ, Wever D, Verlouw AJWE, Nollet $\mathrm{F}$, et al. Risk factors for plantar foot ulcer recurrence in neuropathic diabetic patients. Diabetes Care 2014;37(6):1697-1705. DOI: 10.2337/ dc13-2470.

6. Strandness DE, Priest RE, Gibbons GE. Combined clinical and pathologic study of diabetic and nondiabetic peripheral arterial disease. Diabetes 1964;13(4):366-372. DOI: 10.2337/diab.13.4.366.

7. Mozes G, Keresztury G, Kadar A, Magyar J, Sipos B, Dzsinich S, et al. Atherosclerosis in amputated legs of patients with and without diabetes mellitus. Int Angiol J Int Union Angiol 1998;17(4):282-286.

8. Edmonds ME, Shanahan C, Petrova NL. Diabetic peripheral arteriopathy: a tale of two diseases. In: Piaggesi A, Apelqvist J, Karger SAG, ed. Frontiers in diabetes 2018. pp.60-69. Available from: https:// www.karger.com/Article/FullText/480046.

9. Prompers L, Huijberts M, Apelqvist J, Jude E, Piaggesi A, Bakker K, et al. High prevalence of ischaemia, infection and serious comorbidity in patients with diabetic foot disease in Europe. Baseline results from the eurodiale study. Diabetologia 2007;50(1):18-25. DOI: 10.1007/ s00125-006-0491-1.

10. Giurato L, Meloni M, Izzo V, Uccioli L. Osteomyelitis in diabetic foot: a comprehensive overview. World J Diabetes 2017;8(4):135. DOI: 10.4239/wjd.v8.i4.135.

11. Ragnarson Tennvall G, Apelqvist J. Prevention of diabetes-related foot ulcers and amputations: a cost-utility analysis based on Markov model simulations. Diabetologia 2001;44(11):2077-2087. DOI: 10.1007/ s001250100013.

12. Yazdanpanah L. Literature review on the management of diabetic foot ulcer. World J Diabetes 2015;6(1):37. DOI: 10.4239/wjd.v6.i1.37.

13. Das A, Pendsey S, Abhyankar M, Malabade R. Management of diabetic foot in an Indian clinical setup: an opinion survey. Cureus 2020;12(6):e8636. DOI: 10.7759/cureus.8636Available from: https:// 
www.cureus.com/articles/28674-management-of-diabetic-foot-inan-indian-clinical-setup-an-opinion-survey.

14. Sutkowska E, Sutkowski K, Sokołowski M, Franek E, Dragan S. Distribution of the highest plantar pressure regions in patients with diabetes and its association with peripheral neuropathy, gender, age, and BMI: one centre study. J Diabetes Res 2019;2019:1-11. DOI: $10.1155 / 2019 / 7395769$.
15. Faglia E, Clerici G, Caminiti M, Curci V, Somalvico F. Influence of osteomyelitis location in the foot of diabetic patients with transtibial amputation. Foot Ankle Int 2013;34(2):222-227. DOI: 10.1177/1071100712467436.

16. Cavanagh PR, Bus SA. Off-loading the diabetic foot for ulcer prevention and healing. Plast Reconstr Surg 2011;127:248S-256S. DOI: 10.1097/PRS.0b013e3182024864. 